\title{
GABA-Based Evaluation of Neurologic Conditions: MR Spectroscopy
}

\author{
L.M. Levy and A.J. Degnan
}

\begin{abstract}
SUMMARY: GABA serves as a major neurotransmitter of the brain and functions mainly to inhibit neural excitatory activity. Disruption of the GABAergic processes appears to occur in various neurologic and psychiatric conditions, including epilepsy, mood disorders, motor disorders such as focal dystonia and stiff-person syndrome, sleep disorders, neuroplasticity, and drug and alcohol dependence. These concentration differences may be ascertained by using MR spectroscopy to provide information on the concentration of different metabolites. This review briefly discusses advances in MR spectroscopy methods and explores the application of this technique to detect changes in GABA due to disease processes and medication-induced effects.
\end{abstract}

ABBREVIATIONS: $A E D=$ antiepileptic drug; $G A B A=\gamma$-aminobutyric acid; $\mathrm{GABA}_{\mathrm{A}} \mathrm{R}=\gamma$-amino butyric acid $\mathrm{A}$ receptor; $\mathrm{GABA}_{\mathrm{B}} \mathrm{R}=\gamma$-amino butyric acid $\mathrm{B}$ receptor; GABA-T = GABA transaminase; GAD = glutamic acid decarboxylase; -PRESS = a combined 2D point-resolved spectroscopy sequence; SSRI = selective serotonin reuptake inhibitor

$T^{\mathrm{h}}$ he importance of GABA first emerged from experimental work in the 1950s by Eugene Roberts, identified from extracts of mouse neuroblastoma; the primary inhibitory neurotransmitter activity of GABA was postulated only years after its discovery. ${ }^{1,2}$ GABA is the major inhibitory neurotransmitter within the brain and accounts for almost half of synaptic activity. ${ }^{3}$

GABA arises from glutamic acid by GAD and is broken down by GABA-T. There are 2 major types of GABA receptors: $\mathrm{GABA}_{\mathrm{A}} \mathrm{R}$, which has a predominant inhibitory effect in the cortex, and $\mathrm{GABA}_{\mathrm{B}} \mathrm{R}$, which inhibits excitatory potentials and enables long-term potentiation in the hippocampal and mesolimbic regions. ${ }^{4}$ Alterations in brain GABA concentrations and in GABAergic pathways are implicated in the pathophysiology of a number of neurologic disorders to be discussed in this review.

\section{PRINCIPLES OF GABA MEASUREMENT} ON MR SPECTROSCOPY

Conventional MR spectroscopy techniques can presently assess, with a good degree of reliability, only a small number of metabolites in the human brain. The primary information from the MR spectra has been generally limited to the levels of the few largest

From the Department of Radiology, George Washington University Medical Center, Washington, DC.

Please address correspondence to Lucien M. Levy, MD, Department of Radiology, George Washington University Medical Center, 901 23rd St NW, Washington, DC 20037; e-mail: llevy@mfa.gwu.edu

-- Indicates open access to non-subscribers at www.ajnr.org

http://dx.doi.org/10.3174/ajnr.A2902 peaks: $\mathrm{Cr}$, Cho, NAA, and lactate. GABA appears much lower in concentration, with an average concentration of $1 \mathrm{mmol} / \mathrm{L}$ versus 5-10 mmol/L. ${ }^{5}$ One of the earliest demonstrated detections of GABA in vivo by using MR spectroscopy occurred in the 1990s by Rothman et al. ${ }^{6}$ More recently, additional various technical modifications have been introduced to recognize metabolites such as GABA, glutamine, glutamate, glucose, and others. ${ }^{7}$ These attempts have not always been successful due to difficulties in extracting this type of information from spectra with overlapping resonant peaks.

Another limitation of current MR spectroscopy methods concerns the difficulty of comparing levels of GABA among subjects or in disease states. One way in which researchers have addressed this concern is to express the GABA concentration relative to another metabolite such as Cr. Other problems inherent in MR spectroscopy are the imprecise nature of some ROIs selected to measure metabolites. These ROIs may include differing types of brain matter inadvertently through partial volume effects; gray matter contains a much greater concentration of GABA than white matter. ${ }^{8}$ Moreover, regardless of the size of the ROI, MR spectroscopy cannot indicate whether the concentration of a metabolite measured lies intracellularly or extracellularly; therefore, decreased GABA, for instance, might result from decreased numbers of GABAergic neurons, smaller neurons, or decreased GABA extracellularly. GABA concentrations also may not reflect neurotransmitter flux or the number and activity of GABA receptors. The actual significance of each of these limitations is uncertain at present.

AJNR Am J Neuroradiol 34:259-65 Feb 2013 www.ajnr.org 
Comparison of conventional versus neurotransmitter (GABA) metabolite characteristics for MRS

\begin{tabular}{lcc}
$\begin{array}{c}\text { Metabolite } \\
\text { Characteristics }\end{array}$ & $\begin{array}{c}\text { Conventional } \\
\text { Metabolites } \\
\text { (NAA, Cr, Cho) }\end{array}$ & $\begin{array}{c}\text { Neurotransmitters } \\
\text { (GABA) }\end{array}$ \\
\hline Magnitude & Large & Small \\
Function & Structural & Physiologic \\
Spatial & Homogeneous & Regional \\
Temporal & Constant & Dynamic \\
\hline
\end{tabular}

A comparison of conventional versus neurotransmitter (GABA) metabolite characteristics for MR spectroscopy is shown in the Table. In conventional spectroscopy, the usual metabolites, such as NAA, Cr, and Cho, usually have much greater signal intensity than metabolites such as GABA and other neurotransmitters and are much easier to measure. Also, they tend to vary much less than neurotransmitters temporally and across brain regions. Most important, neurotransmitters are more closely related to brain function, whereas the usual metabolites are more often associated with structural parameters. This becomes important in evaluating various neurologic disorders and the effect of therapies.

\section{Spectral Editing}

Spectral editing sequences have been used to select out J-coupled metabolites from each other; however, only 1 metabolite at a time can be individually selected. A frequency-selective radio-frequency pulse can be applied to generate a spectrum that only depicts signals affected by the selective pulse. ${ }^{9,10}$ Double-quantum filtered and other methods may afford greater editing but may be subject to signal-intensity loss ${ }^{11}$; there are also other spectral editing methods that are beyond the scope of this review. $^{12-18}$

\section{D MR Spectroscopy}

2D proton MR spectroscopy offers the possibility of obtaining all the major cerebral metabolites in vivo in a localized acquisition. ${ }^{19-21}$ Recent techniques to distinguish GABA from other molecules can use spectral editing and 2D uncoupling such as MEGA-PRESS (J-difference editing point resolved magnetic resonance spectroscopy), J-PRESS, and inner volume saturation as exemplified in Fig. 1. ${ }^{22,23}$

\section{CLINICAL USES OF GABA MR SPECTROSCOPY}

Clinically, the ability to obtain cerebral metabolic information provides clinicians with a powerful tool to evaluate or serially follow-up diseases. Present measurements of certain cerebral metabolites, such as GABA, have previously been generally limited to measurements of concentration levels in the blood and CSF, but these are only an indirect reflection of brain levels. Furthermore, they cannot be localized to a particular anatomic region of the brain to assess localized metabolism or regional pathology. Direct MR spectroscopy brain measurements circumvent many of these problems and easily permit serial temporal quantitative assessment of disease progression and drug-mediated effects.
INVESTIGATION OF GABA PHYSIOLOGY BY USING MR SPECTROSCOPY

Recent evidence supports the role of GABA in serving as a gateway for cortical activity. GABA reduction appears to allow increased excitatory activity and vascular reactivity. ${ }^{24-26}$

GABA may also allow synaptic plasticity and cortical reorganization. In a study of deafferentation, Levy et $\mathrm{al}^{27}$ demonstrated rapid decreased GABA concentrations following initiation of a nerve block. This same concept of GABA reduction as an opening of a gate to allow for synaptogenesis is reinforced by experiments of motor learning tasks.

\section{CONDITIONS INVOLVING GABA Epilepsy}

GABA dysfunction has long been postulated as a contributor to seizure activity-reduced GABA would be expected to allow unbridled excitatory neural activity. In line with this theory, antiepileptic medications generally have an effect on increasing GABAergic activity and decreasing CSF levels of GABA. ${ }^{28}$ Moreover, antibodies to GAD have been detected in many groups of patients with seizure disorders; this autoimmune phenomenon would be expected to curtail GABA synthesis. ${ }^{29,30}$ In patients with antiGAD-antibody-positive epilepsy, compared with healthy controls, GABA levels are lower within the primary sensorimotor cortex. ${ }^{31}$ An early study of GABA MR spectroscopy demonstrated that patients with more recent seizure recurrence had much lower concentrations than did those who had been symptom-free longer. ${ }^{32}$ GABA is also reduced in the setting of juvenile myoclonic epilepsy and complex partial seizures. ${ }^{33,34}$ Moreover, poorer seizure control in complex partial seizure disorder appears to correlate with decreased GABA levels. ${ }^{33}$ These findings support the notion of decreased GABAergic function and the occurrence of epilepsy.

On the other hand, others have failed to find decreased GABA concentrations, even following resection of the abnormal seizure focus $^{35}$ or in idiopathic generalized epilepsy while finding elevations in glutamate and glutamine. ${ }^{36,37}$ An abnormal cortex in seizure disorders may demonstrate marked GABA elevation, such as in cortical tubers. ${ }^{38}$ It is clear from these divergent reports that there may be regional variance within different regions of the cortex in different seizure disorders that are still poorly characterized. Ex vivo spectroscopy experiments also contradict these findings, with the detection of increased levels of GABA on MR spectroscopy of brain biopsies from patients with intractable epilepsy. ${ }^{39-41}$

\section{Motor Disorders}

Stiff-person syndrome consists of muscle rigidity and episodic muscle spasms. ${ }^{42}$ As in the case of epilepsy, autoantibodies to the GAD enzyme (GAD-65) have been detected. ${ }^{43-45}$ Reduced CSF GABA levels suggest that decreased inhibitory activity leads to excessive cortical-directed muscle excitation. ${ }^{44}$ On MR spectroscopy, Levy et $\mathrm{al}^{42}$ reported prominent decreases in GABA in the brains of these patients.

Similarly, decreased inhibition by GABA may result in focal dystonia, such as in the case of writer's cramp. ${ }^{46}$ This decrease in 

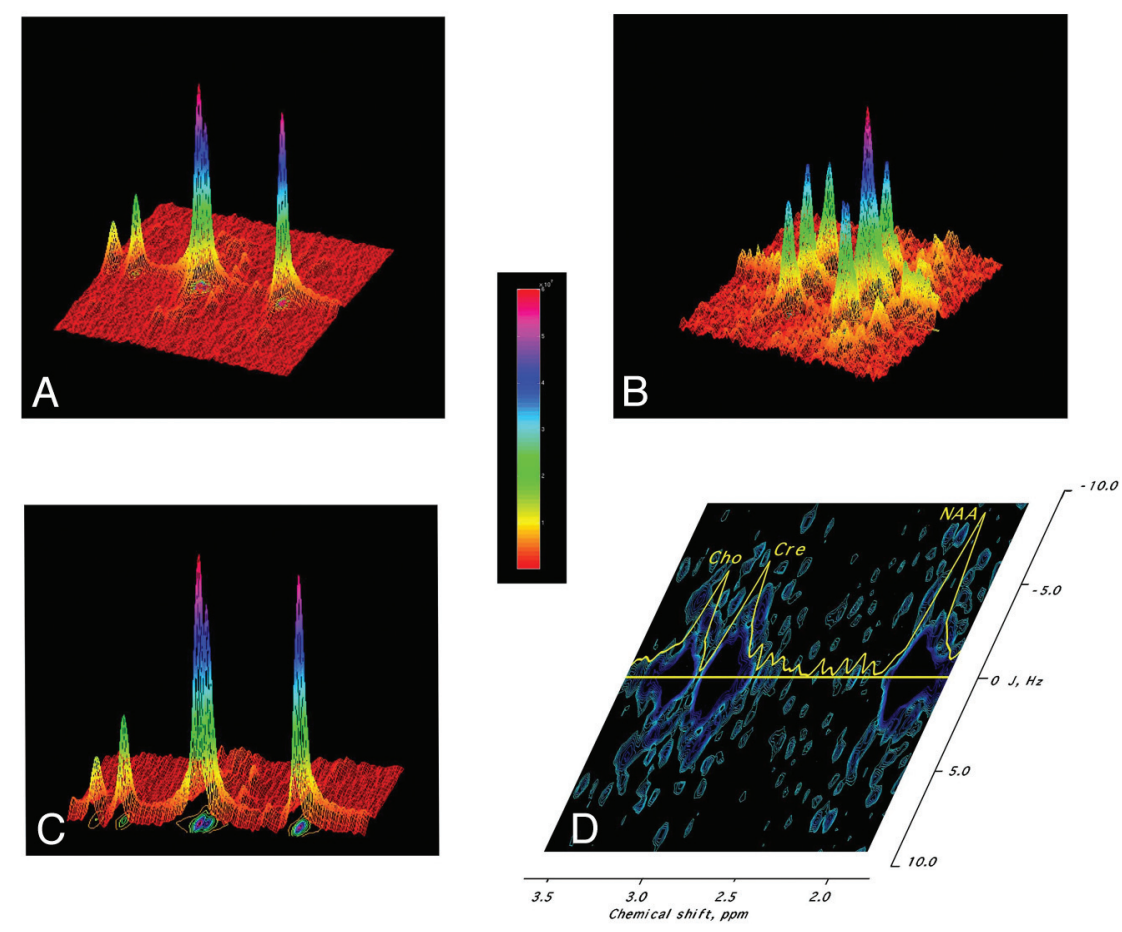

FIG 1. Example of 2D J-PRESS spectroscopy. A, 2D spectrum of a phantom containing NAA and $\mathrm{Cr}$ displayed in 3D, with the vertical axis being signal intensity; the horizontal axis, the chemical shift; and the oblique axis, the "decoupling" or J-dimension. Introduction of this second dimension provides spectra of greater resolution, as if a mountain range was visualized from above (2D spectrum) rather than just from its skyline (conventional ID spectrum). The color bar denotes signal intensity. $B, 2 D$ spectrum of GABA shows the characteristic set of 3 vertical rows compatible with its spectroscopic structure of 2 triplets and 1 multiplet. A large central peak of GABA triplet ( $\alpha$-Carbon Hydrogen $[\mathrm{CH}]$ ) is shown. C, Projection of the 2D spectrum in $A$ maps the individual peaks onto a contour plot. $D, 2 \mathrm{D}$ spectroscopy contour plot of the human sensorimotor cortex. The horizontal axis represents chemical shift; the vertical axis represents the J-axis. The decoupled peaks of the metabolites can then be identified from their known assigned resonances. The ID profile along the J $=0$ axis is plotted in yellow. Large peaks occurring along the $J_{0}$ axis $\left(V_{0}\right.$ subspectrum) at the characteristic chemical shifts of the major peaks of NAA (CH3 at $2.02 \mathrm{ppm}), \mathrm{Cr}(\mathrm{CH} 3$ at $3.04 \mathrm{ppm})$, and $\mathrm{Cho}(\mathrm{CH} 3$ at $3.2 \mathrm{ppm})$ are marked. The central peak of the $\mathrm{GABA}-\mathrm{CH} 2$ resonance can be seen on $J_{0}$ subspectrum (2.31 ppm), with side peaks corresponding to the triplet structure $( \pm 7.3 \mathrm{~Hz})$.

the amount of GABA is thought to encourage plasticity and perhaps lead to excessive plasticity and functional impairment. ${ }^{47}$

\section{Mood and Anxiety Disorders}

Mood disorders also appear to share the phenomenon of reduced GABA. ${ }^{48}$ Patients with unmedicated major depressive disorder have demonstrably lower GABA levels within the dorsomedial/ dorsal anterolateral prefrontal cortex ${ }^{49}$ and occipital cortex. ${ }^{50}$ GABAergic decreases have been noted in depressed subjects in both the occipital and anterior cingulate cortex. ${ }^{51}$ Bipolar patients would be expected to share these brain chemistry changes, especially with demonstrated histologic loss of GABAergic neurons and decreased plasma GABA levels..$^{52,53}$

Angiogenesis may involve GABAergic dysfunction, allowing increased neural excitability as suggested by the effectiveness of benzodiazepines as anxiolytics. Patients with panic disorder possess significantly lower GABA levels than controls. ${ }^{54}$ Those patients on chronic anxiolytics also had decreased GABA concentrations in the occipital cortex and showed blunted responses to benzodiazepines, suggesting impaired GAD function. ${ }^{55}$ On the other hand, patients with social anxiety disorder, while having significantly elevated glutamate and glutamine, showed no differences in GABA compared with healthy controls. ${ }^{56}$

In an elegant threat-of-shock experiment by using MR spectroscopy, Hasler et $\mathrm{al}^{57}$ showed that healthy individuals demonstrate an average $18 \%$ reduction in prefrontal GABA from this acute stress scenario. Because anxiety is closely related to perceived threat, this study suggests that GABA mediates fear-a decrease in GABA might allow priming of a motor response to a feared threat.

\section{Schizophrenia}

It is hypothesized that the cognitive impairments observed in schizophrenia may be related to decreased GABA levels resulting from pancortical decreased GAD transcription. ${ }^{58}$ A recent MR spectroscopy study found that GABA is decreased within the visual cortex in patients with schizophrenia, reinforcing the idea of GABA disruption as the mechanism of loss of cognitive inhibition. ${ }^{59}$ Tayoshi et $\mathrm{al}^{60}$ found more GABA in those patients taking typical antipsychotics versus atypical antipsychotics. Thus, treatment effects may hamper the ability to detect GABA reductions in patients with schizophrenia on medication.

\section{Alcoholism and Drug Addiction}

The importance of GABA in alcoholism is evident in the clinical utility of benzodiazepines in mitigating seizure activity in the setting of acute withdrawal. ${ }^{61}$ Similarly, baclofen (a $\mathrm{GABA}_{\mathrm{B}} \mathrm{R}$ agonist) appears to reduce craving and consumption of alcohol through a GABA-related mechanism. ${ }^{62}$ Alcohol appears to facilitate GABAergic activity at the $\mathrm{GABA}_{\mathrm{A}} \mathrm{R}$; therefore, it is suggested that GABA levels in chronic alcohol use would be lower. ${ }^{61,63}$ MR spectroscopy of alcohol-dependent subjects supports the concept of alcohol-induced GABAergic modification. GABA plus homocarnosine was significantly lower in patients with recently treated alcoholic hepatic encephalopathy and those recently detoxified compared with healthy controls. ${ }^{64}$ In another study, decreased levels of GABA in alcohol-dependent individuals versus healthy controls were not found ${ }^{65}$; this discrepancy may be related to the time course of alcohol withdrawal. In fact, GABA levels were found to be normal shortly after withdrawal and then decreased after a month of sobriety. ${ }^{66}$

Cocaine users share similar findings as those seen in alcoholics: Cerebral GABA levels were significantly reduced in several studies when users were compared with controls. ${ }^{67,68}$ Reductions within the prefrontal cortex explain disinhibition and impaired impulse control seen with cocaine use. 


\section{Sleep Disorders}

GABA has been implicated in sleep disorders, with benzodiazepines having demonstrable effectiveness in treating insomnia. This theory is backed by the finding of substantially reduced

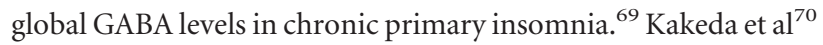
noted a substantial reduction in the GABA/Cr ratio in the frontal lobes of alternate-shift workers by using MR spectroscopy.

Conversely, young patients with narcolepsy with established human leukocyte antigen mutations manifest a completely different appearance on MR spectroscopy, with increases in GABA. ${ }^{71}$ This finding is unsurprising because, in narcolepsy, hypocretin is deficient; hypocretin normally has a negative feedback effect on GABA anabolism. Thus, the elevation of GABA within the medial prefrontal cortex fits into the pathophysiology of narcolepsy.

\section{Migraines}

Bigal et $\mathrm{al}^{72}$ were the first to look at GABA concentrations in migraines by using MR spectroscopy. There was no difference between those with migraines and controls on the basis of GABA values. ${ }^{72}$ Particular to this condition and the concomitant association with neurovascular alterations, GABA changes may simply reflect the effects of altered cerebral blood flow during a migraine attack.

\section{Autism Spectrum Disorders}

$\mathrm{GABA}_{\mathrm{A}}$ and $\mathrm{GABA}_{\mathrm{B}}$ receptor downregulation has been proposed as a potential pathophysiologic mechanism in autism. ${ }^{73,74}$ Harada et $\mathrm{al}^{75}$ recently reported the first MR spectroscopy evaluation of GABA in children with autism, which demonstrated significantly reduced GABA concentration in the frontal lobes and in the GABA/Glu ratio, suggesting that GABAergic activity declines while glutamatergic activity is excessive. These reductions in GABA could explain the cognitive impairment and increased seizure risk inherent in autism.

\section{Olfactory and Gustatory Disorders}

Like the other conditions described herein, the empiric effectiveness of GABA-active medications suggested the role of GABA downregulation in the case of phantogeusia and phantosmia. ${ }^{76}$ In a study of patients with either condition, GABA levels were decreased within the cingulate, right and left insula, and left amygdala. ${ }^{77}$ GABA agonist therapy (haloperidol or thioridazine) significantly increased these depressed GABA levels. ${ }^{77}$ From this clinical research, cortical hyperactivity potentially manifests itself as spurious perceived smells and/or tastes.

\section{TREATMENT EFFECTS ON GABA CONCENTRATION}

MR spectroscopy is emerging as a useful instrument to improve our understanding of the pharmacology and development of medications. ${ }^{65}$ As a noninvasive repeatable study, MR spectroscopy could be easily used to longitudinally follow the changes in brain chemistry. Unexpected changes in brain metabolism could serve as indicators of potential neurologic complications before actual clinical findings appear.

\section{GABA Agonists}

Several medications are known to act either directly on GABA receptors or the related benzodiazepine receptors. Zolpidem, used in the treatment of insomnia and acting on the $\mathrm{GABA}_{\mathrm{A}} \mathrm{R}$, decreased GABA levels in the thalamus but not the anterior cingulate cortex; this effect correlated with subjective nausea and dizziness. ${ }^{78}$ Clonazepam also reduced GABA levels, particularly within the occipital cortex. ${ }^{55}$

\section{ANTI-EPILEPTIC DRUGS \\ Management of Seizure Disorders Relies on the Preventive Function of AEDs.}

Vigabatrin acts by inhibiting GABA-T irreversibly, thereby increasing the amount of GABA available. Early studies showed a significant GABA increase, beginning within 2 hours $^{79,80}$ and verified by others. ${ }^{81,82}$ Increased CSF and MR spectroscopy-measured GABA by vigabatrin are associated with improved seizure control $^{83}$; those with lower baseline levels and steeper increases appear to respond better clinically. ${ }^{84}$

Topiramate is an AED with an imprecise mechanism of action that is thought to enhance $\mathrm{GABA}_{\mathrm{A}}$ activity. Topiramate can increase MR spectroscopy-detected GABA by $72 \%$ or $0.9 \mathrm{mmol} / \mathrm{L}$ in as little as 3-4 hours as well as its precursor, glutamine. ${ }^{85-88}$

Lamotrigine, of the newer generation of AEDs, has proved very useful in other conditions such as bipolar disorder. Despite its efficacy in seizure prevention, ${ }^{89}$ MR spectroscopy showed no GABA effect for lamotrigine acutely but revealed significant GABA increases of one-quarter at 1 month. ${ }^{90}$

Gabapentin is, instead, thought to act via voltage-gated calcium channels. MR spectroscopy illustrates the GABAergic activity of gabapentin, with acute and chronic elevations in $\mathrm{GABA}^{90}$ and a median concentration increase of $1.3 \mathrm{mmol} / \mathrm{L}$ within the occipital cortex within 1 hour of administration. ${ }^{91}$ Levetiracetam is another AED with a tenuous mechanism of action that is thought to bind synaptic vesicles. In patients with epilepsy who responded to levetiracetam, GABA/Cr increased; this change suggests a GABAergic component that facilitates seizure prevention. ${ }^{5}$

\section{Antidepressants}

SSRIs have become the mainstay of the management of mood disorders and related conditions. Given the reduction in GABA seen in depressed patients and the clinical efficacy of SSRIs, it is unsurprising that recent studies suggest that SSRIs increase GABA. A pilot study of several SSRIs showed significantly increased occipital cortex GABA (34\%) after an average of 2 months of treatment. ${ }^{92}$ Citalopram administration yields a $35 \%$ relative increase in GABA in the occipital cortex of healthy volunteers, confirming these findings and suggesting a pro-GABAergic effect of SSRIs. ${ }^{93}$

\section{Nonpharmacologic Therapy}

Because other treatment options may have an effect on the brain chemistry of patients, it is important to systematically assess potential changes in GABA with alternative therapies. Yoga therapy for healthy volunteers yielded demonstrable increases in thalamic GABA on MR spectroscopy over a walking group, ${ }^{94}$ with a significant decrease in anxiety. ${ }^{94}$ On the other hand, GABA MR spectroscopy may not detect changes with some therapies: Cognitive behavioral therapy was of no benefit in increasing GABA levels in individuals with depression in 1 study. ${ }^{95}$ 


\section{FUTURE DIRECTIONS}

MR spectroscopy has already made demonstrable advances in the noninvasive detection of differences in GABA concentrations in a variety of conditions from autism to epilepsy to stiff-person syndrome. In addition, MR spectroscopy informs clinicians of the effects of medications on the chemical composition of the brain. The future of MR spectroscopy looks promising with the advent of advanced techniques that can better isolate individual compounds. Many limitations still exist, however: MR spectroscopy studies are lengthy and uncomfortable for patients, and MR spectroscopy of GABA requires a high degree of technical sophistication. Most pressingly, pervasive gaps in our understanding of neurochemical changes, particularly affecting GABA, persist. Another limitation in the field of MR spectroscopy results from the static approach to metabolic disruptions in the brain. With the use of other MR spectra such as carbon 13 MR spectroscopy, it is plausible that metabolite pool kinetics could be visualized, though several barriers to this approach invented in the 1990s still exist as discussed by others. ${ }^{96,97}$ Future examinations by using MR spectroscopy could take advantage of these novel techniques to examine disruptions of brain chemistry in disease conditions and analyze the effectiveness and action of pharmaceuticals.

\section{REFERENCES}

1. Roberts E, Baxter CF. Metabolic studies of gamma-aminobutyric acid. Neurology 1958;8(suppl 1):77-78

2. Roberts E, Rothstein M, Baxter CF. Some metabolic studies of gamma-aminobutyric acid. Proc Soc Exp Biol Med 1958;97:796-802

3. Defelipe J. Neocortical neuronal diversity: chemical heterogeneity revealed by colocalization studies of classic neurotransmitters, neuropeptides, calcium-binding proteins, and cell-surface molecules. Cerebral Cortex 1993;3:273-89

4. Chang L, Cloak CC, Ernst T. Magnetic resonance spectroscopy studies of GABA in neuropsychiatric disorders. J Clin Psychiatry 2003; 64(suppl 3):7-14

5. Doelken MT, Hammen T, Bogner W, et al. Alterations of intracerebral gamma-aminobutyric acid (GABA) levels by titration with levetiracetam in patients with focal epilepsies. Epilepsia 2010;51: 1477-82

6. Rothman DL, Petroff OA, Behar KL, et al. Localized 1H NMR measurements of gamma-aminobutyric acid in human brain in vivo. Proc Natl Acad Sci U S A 1993;90:5662-66

7. Bonavita S, Di Salle F, Tedeschi G. Proton MRS in neurological disorders. Eur J Radiol 1999;30:125-31

8. Goto N, Yoshimura R, Moriya J, et al. Critical examination of a correlation between brain gamma-aminobutyric acid (GABA) concentrations and a personality trait of extroversion in healthy volunteers as measured by a 3 Tesla proton magnetic resonance spectroscopy study. Psychiatry Res 2010;182:53-57

9. Jensen JE, Frederick BD, Wang L, et al. Two-dimensional, J-resolved spectroscopic imaging of GABA at 4 Tesla in the human brain. Magn Reson Med 2005;54:783-88

10. Jensen JE, Frederick Bde B, Renshaw PF. Grey and white matter GABA level differences in the human brain using two-dimensional, J-resolved spectroscopic imaging. NMR Biomed 2005; 18:570-76

11. Keltner JR, Wald LL, Frederick BD, et al. In vivo detection of GABA in human brain using a localized double-quantum filter technique. Magn Reson Med 1997;37:366-71

12. Choi C, Bhardwaj PP, Kalra S, et al. Measurement of GABA and contaminants in gray and white matter in human brain in vivo. Magn Reson Med 2007;58:27-33

13. Choi IY, Lee SP, Shen J. In vivo single-shot three-dimensionally localized multiple quantum spectroscopy of GABA in the human brain with improved spectral selectivity. J Magn Reson 2005; 172:9-16

14. Choi IY, Lee SP, Merkle H, et al. Single-shot two-echo technique for simultaneous measurement of GABA and creatine in the human brain in vivo. Magn Reson Med 2004;51:1115-21

15. Snyder J, Hanstock CC, Wilman AH. Spectral editing of weakly coupled spins using variable flip angles in PRESS constant echo time difference spectroscopy: application to GABA. J Magn Reson 2009;200:245-50

16. Shen J. Slice-selective J-coupled coherence transfer using symmetric linear phase pulses: applications to localized GABA spectroscopy. J Magn Reson 2003;163:73-80

17. Waddell KW, Avison MJ, Joers JM, et al. A practical guide to robust detection of GABA in human brain by J-difference spectroscopy at 3 T using a standard volume coil. Magn Reson Imaging 2007;25:103238. Epub 2007 Feb 2

18. Henry ME, Lauriat TL, Shanahan M, et al. Accuracy and stability of measuring GABA, glutamate, and glutamine by proton magnetic resonance spectroscopy: a phantom study at 4 Tesla. J Magn Reson 2011;208:210-18

19. Ke Y, Cohen BM, Bang JY, et al. Assessment of GABA concentration in human brain using two-dimensional proton magnetic resonance spectroscopy. Psychiatry Res 2000;100:169-78

20. Ryner LN, Sorenson JA, Thomas MA. 3D localized 2D NMR-spectroscopy on an MRI scanner. J Magn Reson Series B 1995;107:126-37

21. Ryner LN, Sorenson JA, Thomas MA. Localized 2D J-resolved $1 \mathbf{H}$ MR spectroscopy: strong coupling effects in vitro and in vivo. Magn Reson Imaging 1995;13:853-69

22. Edden RA, Barker PB. Spatial effects in the detection of gammaaminobutyric acid: improved sensitivity at high fields using inner volume saturation. Magn Reson Med 2007;58:1276-82

23. Edden RA, Barker PB. If J doesn't evolve, it won't J-resolve: J-PRESS with bandwidth-limited refocusing pulses. Magn Reson Med 2011;65:1509-14

24. Donahue MJ, Near J, Blicher JU, et al. Baseline GABA concentration and fMRI response. Neuroimage 2010; 53:392-98

25. Northoff G, Walter M, Schulte RF, et al. GABA concentrations in the human anterior cingulate cortex predict negative BOLD responses in fMRI. Nat Neurosci 2007;10:1515-17

26. Muthukumaraswamy SD, Edden RA, Jones DK, et al. Resting GABA concentration predicts peak gamma frequency and $\mathrm{fMRI}$ amplitude in response to visual stimulation in humans. Proc Natl Acad Sci US A 2009;106:8356-61

27. Levy LM, Ziemann U, Chen R, et al. Rapid modulation of GABA in sensorimotor cortex induced by acute deafferentation. Ann Neurol 2002;52:755-61

28. Yamamoto M, Takahashi S, Otsuki S, et al. GABA levels in cerebrospinal fluid of patients with epilepsy. Folia Psychiatr Neurol Jpn 1985;39:515-19

29. McKnight K, Jiang Y, Hart Y, et al. Serum antibodies in epilepsy and seizure-associated disorders. Neurology 2005;65:1730-36

30. Peltola J, Kulmala P, Isojarvi J, et al. Autoantibodies to glutamic acid decarboxylase in patients with therapy-resistant epilepsy. Neurology 2000;55:46-50

31. Stagg CJ, Lang B, Best JG, et al. Autoantibodies to glutamic acid decarboxylase in patients with epilepsy are associated with low cortical GABA levels. Epilepsia 2010;51:1898-901

32. Petroff OA, Rothman DL, Behar KL, et al. Low brain GABA level is associated with poor seizure control. Ann Neurol 1996;40:908-11

33. Petroff OA, Hyder F, Rothman DL, et al. Homocarnosine and seizure control in juvenile myoclonic epilepsy and complex partial seizures. Neurology 2001;56:709-15

34. Petroff OA, Behar KL, Rothman DL. New NMR measurements in epilepsy: measuring brain GABA in patients with complex partial seizures. Adv Neurol 1999;79:939-45

35. Simister RJ, McLean MA, Barker GJ, et al. Proton MR spectroscopy of metabolite concentrations in temporal lobe epilepsy and effect of temporal lobe resection. Epilepsy Res 2009;83:168-76 
36. Simister RJ, McLean MA, Barker GJ, et al. Proton MRS reveals frontal lobe metabolite abnormalities in idiopathic generalized epilepsy. Neurology 2003;61:897-902

37. Simister RJ, McLean MA, Barker GJ, et al. Proton magnetic resonance spectroscopy of malformations of cortical development causing epilepsy. Epilepsy Res 2007;74:107-15

38. Taki MM, Harada M, Mori K, et al. High gamma-aminobutyric acid level in cortical tubers in epileptic infants with tuberous sclerosis complex measured with the MEGA-editing J-difference method and a three-Tesla clinical MRI instrument. Neuroimage 2009;47: 1207-14

39. Aasly J, Silfvenius H, Aas TC, et al. Proton magnetic resonance spectroscopy of brain biopsies from patients with intractable epilepsy. Epilepsy Res 1999;35:211-17

40. Petroff OA, Pleban LA, Spencer DD. Symbiosis between in vivo and in vitro NMR spectroscopy: the creatine, $\mathrm{N}$-acetylaspartate, glutamate, and GABA content of the epileptic human brain. Magn Reson Imaging 1995;13:1197-211

41. Petroff OA, Rothman DL, Behar KL, et al. Initial observations on effect of vigabatrin on in vivo $1 \mathrm{H}$ spectroscopic measurements of gamma-aminobutyric acid, glutamate, and glutamine in human brain. Epilepsia 1995; 36:457-64

42. Levy LM, Levy-Reis I, Fujii M, et al. Brain gamma-aminobutyric acid changes in stiff-person syndrome. Arch Neurol 2005;62:970-74

43. Dalakas MC, Fujii M, Li M, et al. The clinical spectrum of anti-GAD antibody-positive patients with stiff-person syndrome. Neurology 2000; 55:1531-35

44. Dalakas MC, Li M, Fujii M, et al. Stiff person syndrome: quantification, specificity, and intrathecal synthesis of GAD65 antibodies. Neurology 2001;57:780-84

45. Murinson BB, Butler M, Marfurt K, et al. Markedly elevated GAD antibodies in SPS: effects of age and illness duration. Neurology 2004;63:2146-48

46. Levy LM, Hallett M. Impaired brain GABA in focal dystonia. Ann Neurol 2002;51:93-101

47. Quartarone A, Siebner HR, Rothwell JC. Task-specific hand dystonia: can too much plasticity be bad for you? Trends Neurosci 2006;29:192-99

48. Taylor M, Bhagwagar Z, Cowen PJ, et al. GABA and mood disorders. Psychol Med 2003;33:387-93

49. Hasler G, van der Veen JW, Tumonis T, et al. Reduced prefrontal glutamate/glutamine and gamma-aminobutyric acid levels in major depression determined using proton magnetic resonance spectroscopy. Arch Gen Psychiatry 2007;64:193-200

50. Sanacora G, Mason GF, Rothman DL, et al. Reduced cortical gamma-aminobutyric acid levels in depressed patients determined by proton magnetic resonance spectroscopy. Arch Gen Psychiatry 1999;56:1043-47

51. Bhagwagar Z, Wylezinska M, Jezzard P, et al. Low GABA concentrations in occipital cortex and anterior cingulate cortex in medication-free, recovered depressed patients. Int J Neuropsychopharmacol 2008;11:255-60

52. Cotter D, Landau S, Beasley C, et al. The density and spatial distribution of GABAergic neurons, labelled using calcium binding proteins, in the anterior cingulate cortex in major depressive disorder, bipolar disorder, and schizophrenia. Biol Psychiatry 2002;51: $377-86$

53. Berrettini WH, Nurnberger JI Jr, Hare TA, et al. Reduced plasma and CSF gamma-aminobutyric acid in affective illness: effect of lithium carbonate. Biol Psychiatry 1983;18:185-94

54. Goddard AW, Mason GF, Almai A, et al. Reductions in occipital cortex GABA levels in panic disorder detected with $1 \mathrm{H}$-magnetic resonance spectroscopy. Arch Gen Psychiatry 2001;58:556-61

55. Goddard AW, Mason GF, Appel M, et al. Impaired GABA neuronal response to acute benzodiazepine administration in panic disorder. Am J Psychiatry 2004;161:2186-93

56. Pollack MH, Jensen JE, Simon NM, et al. High-field MRS study of GABA, glutamate and glutamine in social anxiety disorder: re- sponse to treatment with levetiracetam. Prog Neuropsychopharmacol Biol Psychiatry 2008;32:739-43

57. Hasler G, van der Veen JW, Grillon C, et al. Effect of acute psychological stress on prefrontal GABA concentration determined by proton magnetic resonance spectroscopy. Am J Psychiatry 2010; 167:1226-31

58. Hashimoto T, Bazmi HH, Mirnics K, et al. Conserved regional patterns of GABA-related transcript expression in the neocortex of subjects with schizophrenia. Am J Psychiatry 2008;165:479-89

59. Yoon JH, Maddock RJ, Rokem A, et al. GABA concentration is reduced in visual cortex in schizophrenia and correlates with orientation-specific surround suppression. J Neurosci 2010;30:3777-81

60. Tayoshi S, Nakataki M, Sumitani S, et al. GABA concentration in schizophrenia patients and the effects of antipsychotic medication: a proton magnetic resonance spectroscopy study. Schizophr Res 2010;117:83-91

61. Krystal JH, Staley J, Mason G, et al. Gamma-aminobutyric acid type A receptors and alcoholism: intoxication, dependence, vulnerability, and treatment. Arch Gen Psychiatry 2006;63:957-68

62. Addolorato G, Caputo F, Capristo E, et al. Baclofen efficacy in reducing alcohol craving and intake: a preliminary double-blind randomized controlled study. Alcohol Alcohol 2002; 37:504-08

63. Ticku MK. Alcohol and GABA-benzodiazepine receptor function. Ann Med 1990;22:241-46

64. Behar KL, Rothman DL, Petersen KF, et al. Preliminary evidence of low cortical GABA levels in localized 1H-MR spectra of alcoholdependent and hepatic encephalopathy patients. Am J Psychiatry 1999; 156:952-54

65. Mason GF, Petrakis IL, de Graaf RA, et al. Cortical gamma-aminobutyric acid levels and the recovery from ethanol dependence: preliminary evidence of modification by cigarette smoking. Biol Psychiatry 2006;59:85-93

66. Mason G, Appel M, de Graaf R, et al. Brain GABA falls by 1 month of detoxification and remains low. Alcohol Clin Exp Res 2003 Suppl(27):56A

67. Ke Y, Streeter CC, Nassar LE, et al. Frontal lobe GABA levels in cocaine dependence: a two-dimensional, J-resolved magnetic resonance spectroscopy study. Psychiatry Res 2004;130:283-93

68. Ke Y, Streeter CC, Yurgelun-Todd DA, et al. A J-resolved, two dimensional MRS study of brain GABA and NAA levels in cocaine dependent subjects-before and after treatment. In: Proceedings of the International Society of Magnetic Resonance in Medicine, May 7-13, 2001; Montreal, Quebec, Canada

69. Winkelman JW, Buxton OM, Jensen JE, et al. Reduced brain GABA in primary insomnia: preliminary data from $4 \mathrm{~T}$ proton magnetic resonance spectroscopy (1H-MRS). Sleep 2008;31:1499-506

70. Kakeda S, Korogi Y, Moriya J, et al. Influence of work shift on glutamic acid and gamma-aminobutyric acid (GABA): evaluation with proton magnetic resonance spectroscopy at 3T. Psychiatry Res 2011;192:55-59

71. Kim SJ, Lyoo IK, Lee YS, et al. Increased GABA levels in medial prefrontal cortex of young adults with narcolepsy. Sleep 2008;31:342-47

72. Bigal ME, Hetherington $\mathrm{H}, \mathrm{Pan} \mathrm{J}$, et al. Occipital levels of GABA are related to severe headaches in migraine. Neurology 2008;70: 2078-80

73. Fatemi SH, Folsom TD, Reutiman TJ, et al. Expression of GABA(B) receptors is altered in brains of subjects with autism. Cerebellum 2009;8:64-69

74. Fatemi SH, Reutiman TJ, Folsom TD, et al. GABA(A) receptor downregulation in brains of subjects with autism. J Autism Dev Disord 2009;39:223-30

75. Harada M, Taki MM, Nose A, et al. Non-invasive evaluation of the GABAergic/glutamatergic system in autistic patients observed by MEGA-editing proton MR spectroscopy using a clinical 3T instrument. J Autism Dev Disord 2011;41:447-54

76. Henkin RI, Levy LM, Lin CS. Taste and smell phantoms revealed by 
brain functional MRI (fMRI). J Comput Assist Tomogr 2000;24: $106-23$

77. Levy LM, Henkin RI. Brain gamma-aminobutyric acid levels are decreased in patients with phantageusia and phantosmia demonstrated by magnetic resonance spectroscopy. J Comput Assist Tomogr 2004;28:721-27

78. Licata SC, Jensen JE, Penetar DM, et al. A therapeutic dose of zolpidem reduces thalamic GABA in healthy volunteers: a proton MRS study at 4 T. Psychopharmacology (Berl) 2009;203:819-29. Epub 2009 Jan 6

79. Mattson RH, Petroff OA, Rothman D, et al. Vigabatrin: effect on brain GABA levels measured by nuclear magnetic resonance spectroscopy. Acta Neurol Scand Suppl 1995;162:27-30

80. Petroff OA, Rothman DL, Behar KL, et al. Human brain GABA levels rise rapidly after initiation of vigabatrin therapy. Neurology 1996;47:1567-71

81. Weber OM, Verhagen A, Duc CO, et al. Effects of vigabatrin intake on brain GABA activity as monitored by spectrally edited magnetic resonance spectroscopy and positron emission tomography. Magn Reson Imaging 1999;17:417-25

82. Verhoeff NP, Petroff OA, Hyder F, et al. Effects of vigabatrin on the GABAergic system as determined by [123I]iomazenil SPECT and GABA MRS. Epilepsia 1999;40:1433-38

83. Petroff $\mathrm{OA}$, Rothman DL. Measuring human brain GABA in vivo: effects of GABA-transaminase inhibition with vigabatrin. $\mathrm{Mol} \mathrm{Neu}$ robiol 1998;16:97-121

84. Mueller SG, Weber OM, Duc CO, et al. Effects of vigabatrin on brain GABA+/CR signals in patients with epilepsy monitored by $1 \mathrm{H}-\mathrm{NMR}$ spectroscopy: responder characteristics. Epilepsia 2001;42:29-40

85. Kuzniecky R, Hetherington H, Ho S, et al. Topiramate increases cerebral GABA in healthy humans. Neurology 1998;51:627-29

86. Moore CM, Wardrop M, deB Frederick B, et al. Topiramate raises anterior cingulate cortex glutamine levels in healthy men; a 4.0 T magnetic resonance spectroscopy study. Psychopharmacology (Berl) 2006; 188:236-43
87. Petroff OA, Hyder F, Rothman DL, et al. Topiramate rapidly raises brain GABA in epilepsy patients. Epilepsia 2001;42:543-48

88. Petroff OA, Hyder F, Mattson RH, et al. Topiramate increases brain GABA, homocarnosine, and pyrrolidinone in patients with epilepsy. Neurology 1999;52:473-78

89. Shiah IS, Yatham LN, Gau YC, et al. Effect of lamotrigine on plasma GABA levels in healthy humans. Prog Neuropsychopharmacol Biol Psychiatry 2003;27:419-23

90. Kuzniecky R, Ho S, Pan J, et al. Modulation of cerebral GABA by topiramate, lamotrigine, and gabapentin in healthy adults. Neurology 2002;58:368-72

91. Petroff OA, Hyder F, Rothman DL, et al. Effects of gabapentin on brain GABA, homocarnosine, and pyrrolidinone in epilepsy patients. Epilepsia 2000;41:675-80

92. Sanacora G, Mason GF, Rothman DL, et al. Increased occipital cortex GABA concentrations in depressed patients after therapy with selective serotonin reuptake inhibitors. Am J Psychiatry 2002;159: 663-65

93. Bhagwagar Z, Wylezinska $M$, Taylor $M$, et al. Increased brain GABA concentrations following acute administration of a selective serotonin reuptake inhibitor. Am J Psychiatry 2004;161:368-70

94. Streeter CC, Whitfield TH, Owen L, et al. Effects of yoga versus walking on mood, anxiety, and brain GABA levels: a randomized controlled MRS study. J Altern Complement Med 2010;16:1145-52

95. Sanacora G, Gueorguieva R, Epperson CN, et al. Subtype-specific alterations of gamma-aminobutyric acid and glutamate in patients with major depression. Arch Gen Psychiatry 2004;61:705-13

96. Manor D, Rothman DL, Mason GF, et al. The rate of turnover of cortical GABA from [1-C-13] glucose is reduced in rats treated with the GABA-transaminase inhibitor vigabatrin (gamma-vinyl GABA). Neurochem Res 1996;21:1031-41

97. Dager SR, Corrigan NM, Richards TL, et al. Research applications of magnetic resonance spectroscopy to investigate psychiatric disorders. Top Magn Reson Imaging 2008;19:81-96 